\title{
Strides in trauma care
}

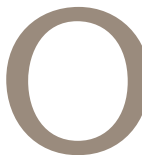

$\mathrm{n}$ very, very few occasions is progress made literally overnight with an entire national healthcare programme. I have never heard of a cost reduction programme successfully rolled out over a nation's healthcare system that has resulted in tangibly improved care and patient outcomes.

This is exactly what the NHS attempted to do on the 1st April 2012. In probably what has been the biggest experiment in healthcare, the management of badly injured patients across the nation changed overnight. A year on, the first report of the national trauma system in England and Wales has emerged, and the headline figures from the national trauma audit network (TARN) have revealed a $20 \%$ improvement in survival following major injury. Amazingly this new system is cheaper and demonstrates how the reconfiguration and redesign of existing services can be achieved successfully, while providing clear improvement of patient outcomes on a local and national level. There are few national initiatives that have actually had this desired effect. The introduction of the independent sector treatment centres to improve 'productivity' in joint replacement has been met with, at best, suspicion, and in some cases there has been cause for significant clinical or efficiency concern. I wonder if taking the same top down restructuring coupled with best practice incentives would be a much more sensible approach to improve outcomes and productivity in other areas of orthopaedics such as a joint replacement, specifically, and surgery as a whole.

This month in 360 we share a fascinating article on the history of arthroscopy from Chuck Cakic which traces the evolution of a completely new surgical approach and technique from its earliest beginnings to what it has become today. This discusses the contributions of the early innovators in the field, from the use of Hopkins rod telescopes for diagnostic knee arthroscopy to the most innovative current shoulder arthroscopic techniques. I hope you will enjoy reading the (relatively recent) history of what has become an established orthopaedic staple. In a complimentary Technique in Focus article, Graham Tytherleigh-Strong describes in more detail his pioneering technique for diagnostic and therapeutic arthroscopy of the sternoclavicular joint.'

One of the biggest challenges facing surgeons, scientists and to some extent patients is the widening evidence gap. While pharmaceutical companies lend themselves to a more traditional evidence based medicine approach with evaluation through randomised controlled trials; more complex interventions (chiefly surgery, but also interventional radiology, physiotherapy etc) do not. The 'IDEAL' group have outlined a comprehensive approach to deal with this and I would recommend the series of articles in the British Medical Journal2-4 to all our readers. Other highlights, for me as Editor, in this edition are the opinion pieces from the New England Journal of Medicine describing the medical response to the April 2013 Boston marathon bombings. ${ }^{5,6}$

My very best wishes to you all.

\section{REFERENCES}

1. Tytherleigh-Strong G. Arthroscopy of the sternoclavicular joint. Arthrosc Tech 2013;2:141-145.

2. McCulloch P, Cook JA, Altman DG, et al. IDEAL framework for surgical innovation 1: the idea and development stages. BMJ 2013;346: $\{3012$.

3. Ergina PL, Barkun JS, McCulloch P, et al. IDEAL framework for surgical innovation 2: observational studies in the exploration and assessment stages. BMJ 2013;346:f3011.

4. Cook JA, McCulloch P, Blazeby JM, et al. IDEAL framework for surgical innovation 3: randomised controlled trials in the assessment stage and evaluations in the long term study stage. BMJ 2013;346:f2820. 5. Biddinger PD, Baggish A, Harrington L, et al. Be prepared: the Boston marathon and mass-casualty events. $N$ Engl J Med 2013;368:1958-1960.

6. Jangi S. Under the medical tent at the Boston marathon. $N$ Eng/J Med 2013;368:1953-1955. 